\title{
Associations of Skeletal Muscle With Symptom Burden and Clinical Outcomes in Hospitalized Patients With Advanced Cancer
}

\author{
Emily van Seventer, $\mathrm{MPH}^{1, *}$; J. Peter Marquardt'2,*; Amelie S. Troschel2; Till D. Best ${ }^{2,3}$; Nora Horick, MS ; \\ Chinenye Azoba, BS ${ }^{1}$; Richard Newcomb, MD'1; Eric J. Roeland, MD ${ }^{1}$; Michael Rosenthal, MD, PhD ${ }^{5,6}$;

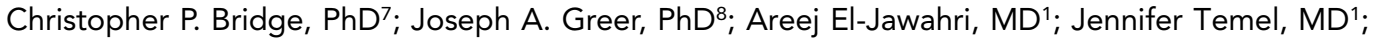 \\ Florian J. Fintelmann, $\mathrm{MD}^{2,+}$; and Ryan D. Nipp, MD, MPH ${ }^{1,+}$
}

\begin{abstract}
Background: Low muscle mass (quantity) is common in patients with advanced cancer, but little is known about muscle radiodensity (quality). We sought to describe the associations of muscle mass and radiodensity with symptom burden, healthcare use, and survival in hospitalized patients with advanced cancer. Methods: We prospectively enrolled hospitalized patients with advanced cancer from September 2014 through May 2016. Upon admission, patients reported their physical (Edmonton Symptom Assessment System [ESAS]) and psychological (Patient Health Questionnaire-4 [PHQ-4]) symptoms. We used CT scans performed per routine care within 45 days before enrollment to evaluate muscle mass and radiodensity. We used regression models to examine associations of muscle mass and radiodensity with patients' symptom burden, healthcare use (hospital length of stay and readmissions), and survival. Results: Of 1,121 patients enrolled, 677 had evaluable muscle data on CT (mean age, $62.86 \pm 12.95$ years; $51.1 \%$ female). Older age and female sex were associated with lower muscle mass (age: $B,-0.16 ; P<.001$; female: $B,-6.89 ; P<.001$ ) and radiodensity (age: $B,-0.33 ; P<.001$; female: $B,-1.66 ; P=.014$ ), and higher $B M l$ was associated with higher muscle mass $(B, 0.58 ; P<.001)$ and lower radiodensity $(B,-0.61 ; P<.001)$. Higher muscle mass was significantly associated with improved survival (hazard ratio, $0.97 ; P<.001$ ). Notably, higher muscle radiodensity was significantly associated with lower ESAS-Physical ( $B,-0.17 ; P=.016)$, ESAS-Total ( $B$, $-0.29 ; P=.002)$, PHQ-4-Depression $(B,-0.03 ; P=.006)$, and PHQ4-Anxiety $(B,-0.03 ; P=.008)$ symptoms, as well as decreased hospital length of stay $(B,-0.07 ; P=.005)$, risk of readmission or death in 90 days (odds ratio, $0.97 ; P<.001$ ), and improved survival (hazard ratio, $0.97 ; P<.001$ ). Conclusions: Although muscle mass (quantity) only correlated with survival, we found that muscle radiodensity (quality) was associated with patients' symptoms, healthcare use, and survival. These findings underscore the added importance of assessing muscle quality when seeking to address adverse muscle changes in oncology.
\end{abstract}

J Natl Compr Canc Netw 2021;19(3):319-327 doi: 10.6004/jnccn.2020.7618

See JNCCN.org for supplemental online content.

\section{Background}

Patients with cancer often experience loss of skeletal muscle, which adversely impacts clinical outcomes. ${ }^{1,2}$ Although most patients with cancer experience some degree of muscle loss, severe muscle loss contributes to approximately $20 \%$ of all cancer deaths. ${ }^{3-5}$ Notably, patients who meet criteria for sarcopenia (ie, low muscle mass) experience poor treatment tolerability and decreased survival. ${ }^{6-10}$ Although prior studies have described the adverse effects of low muscle mass (ie, muscle quantity), little is known about low muscle radiodensity, or attenuation (ie, muscle quality). Muscle radiodensity provides information about muscle quality by describing fatty infiltration in the skeletal muscle tissue (ie, myosteatosis). ${ }^{11,12}$ To more comprehensively evaluate patients' muscle health, researchers must consider both muscle quantity and quality. ${ }^{13-15}$ The few studies of muscle quality to date suggest that low muscle radiodensity correlates with worsening physical function and increased mortality. ${ }^{2,13}$ However, data are lacking to understand how patients' muscle mass and radiodensity are associated with their symptom burden and healthcare utilization.

Hospitalized patients with advanced cancer represent a group particularly at risk for experiencing high

\footnotetext{
${ }^{1}$ Department of Medicine, Division of Hematology and Oncology, Massachusetts General Hospital Cancer Center and Harvard Medical School, and ${ }^{2}$ Department of Radiology, Massachusetts General Hospital and Harvard Medical School, Boston, Massachusetts; ${ }^{3}$ Department of Radiology, Charité-Universitätsmedizin Berlin, Berlin, Germany; and ${ }^{4}$ Department of Statistics, Massachusetts General Hospital and Harvard Medical School, ${ }^{5}$ Dana-Farber Cancer Institute, ${ }^{6}$ Department of Radiology, Brigham and Women's Hospital, ${ }^{7}$ Massachusetts General Hospital and Brigham and Women's Hospital Center for Clinical Data Science, and ${ }^{8}$ Department of Psychiatry, Massachusetts General Hospital and Harvard Medical School, Boston, Massachusetts.
}

*These authors are co-first authors.

tThese authors are co-last authors. 
symptom burden and loss of muscle. ${ }^{16-18}$ Patients with muscle loss often require hospital-level care to address treatment adverse effects and high symptom burden (eg, pain, fatigue, nausea). ${ }^{19}$ Notably, hospitalizations may further decrease patients' functional ability and physical activity, thereby exacerbating muscle loss. ${ }^{20-22}$ Limited research among patients with cancer in outpatient settings suggests a potential correlation between the severity of muscle loss and greater symptom burden. ${ }^{9,23-25}$ However, we lack information about these outcomes in hospitalized patients. In addition, despite evidence linking patient's symptoms with greater healthcare use, ${ }^{26-30}$ studies investigating relationships among muscle health, symptom burden, and clinical outcomes in patients with advanced cancer are lacking. ${ }^{17,28,31}$ Thus, research is needed to understand these relationships, especially in the uniquely high-risk population of hospitalized patients with advanced cancer.

In this study, we sought to investigate relationships among muscle (mass and radiodensity), patientreported outcomes (physical and psychologic symptoms), and clinical outcomes (healthcare use and survival) in a large cohort of hospitalized patients with advanced cancer. Specifically, we used muscle measurements on CT scans collected as part of routine care to examine associations between patients' muscle health and their physical and psychological symptom burden, healthcare use (hospital length of stay [LOS] and readmissions), and survival. We hypothesized that higher muscle mass and radiodensity would correlate with lower symptom burden and better clinical outcomes. An improved understanding of these relationships could inform future interventions targeting modifiable risk factors, such as symptom burden and adverse muscle changes, and thereby enhance care outcomes for this high-risk cancer population.

\section{Methods}

\section{Study Procedures}

We enrolled patients with advanced cancer who had an unplanned hospitalization at Massachusetts General Hospital from September 2014 through May 2016 in a prospective cohort study. We recruited consecutive patients during their first unplanned hospital admission within the study period by screening the daily inpatient oncology census, and each participant contributed one unique hospitalization. Upon admission (within 5 days of hospitalization), we obtained informed consent and asked patients to complete a symptom burden questionnaire. ${ }^{16,17,28,32,33}$ For this study, we used body composition data obtained from routine clinical CT scans. ${ }^{34,35}$ The Dana-Farber/Harvard Cancer Center
Institutional Review Board approved this study (body composition analysis was conducted as part of a secondary analysis, and the board waived the need to obtain additional consent to access CT scans).

\section{Participants}

Patients were eligible for study participation if they had an unplanned hospital admission at Massachusetts General Hospital, were aged $\geq 18$ years, and had a known diagnosis of incurable cancer. We identified patients with incurable cancer as those being treated with palliative intent per chemotherapy order entry (palliative vs curative) and used oncology note documentation for those not receiving chemotherapy. Study participants had to be able to read and respond to study questionnaires in English or with minimal help from an interpreter. We excluded patients whose hospital admission was planned and/or elective, including hospitalizations for chemotherapy administration/ desensitization and scheduled procedures. We limited the current analysis to participants with a routine CT scan obtained within 45 days before study enrollment that imaged muscle at the level of the third lumbar vertebral body (L3).

\section{Study Measures}

\section{Demographics and Clinical Information}

We obtained demographic information, including date of birth, sex, race, education level, relationship status, and insurance type, by reviewing participants' electronic health records (EHRs). We abstracted height, weight, body mass index (BMI), and Charlson comorbidity index (CCI) score at admission; date of cancer diagnosis; and cancer type from the EHR, which included documentation by the oncology team in the progress notes.

\section{Skeletal Muscle}

We evaluated skeletal muscle using CT scans performed as part of routine clinical care within 45 days before study enrollment. We used a previously described body composition analysis pipeline based on proprietary machine learning algorithms to automatically segment muscle and adipose tissue at L3. ${ }^{34}$ Briefly, the algorithm is a convolutional neural network that was developed on manually segmented CT images at L3 using an attenuation range of -29 to +150 Hounsfield units (HU) for skeletal muscle and an attenuation range of -190 to $-30 \mathrm{HU}$ for adipose tissue (subcutaneous and visceral). In a validation experiment on a different dataset of segmented L3 CT images, the body composition analysis pipeline achieved a high correlation. ${ }^{34}$ In the present study, trained researchers blinded to clinical outcomes 
manually reviewed segmentation quality and removed scans without usable muscle data. We calculated muscle mass (normalized to height, also known as the skeletal muscle index) by dividing the cross-sectional muscle area at $\mathrm{L} 3$ by the patient's squared height $\left(\mathrm{cm}^{2} / \mathrm{m}^{2}\right)$. We categorized patients as sarcopenic based on previously defined cutoff values ( $<39 \mathrm{~cm}^{2} / \mathrm{m}^{2}$ for women, $<55 \mathrm{~cm}^{2}$ ) $\mathrm{m}^{2}$ for men). ${ }^{25}$ We calculated muscle radiodensity as the mean compartment attenuation across all voxels classified as muscle (in HU; Figure 1). We assessed whether patients received intravenous contrast for CT scans, because this could potentially influence muscle measurements, and adjusted for this possibility in statistical analyses. $^{36}$

\section{Symptom Burden}

We assessed patients' symptoms using the selfadministered, revised Edmonton Symptom Assessment System (ESAS-r). ${ }^{37-39}$ The ESAS-r evaluates pain, fatigue, drowsiness, nausea, appetite, dyspnea, depression, anxiety, and well-being over the previous 24 hours. In addition, we included constipation, which commonly occurs in patients with cancer. ${ }^{40}$ Each symptom is individually scored from 0 to 10 , with higher scores indicating greater severity. We calculated the ESAS-Total score as the sum of all ESAS symptoms (score range, 0-100), and computed the ESAS-Physical score as the composite of pain, fatigue, drowsiness, nausea, appetite, dyspnea, and constipation (score range, 0-70). We assessed patients' psychological symptoms using the Patient Health Questionnaire-4 (PHQ-4). ${ }^{41}$ The PHQ-4 consists of 4 questions, with 2 evaluating depression symptoms (PHQ-4-Depression) and 2 evaluating anxiety symptoms (PHQ-4-Anxiety). Higher scores on the PHQ-4-Depression and PHQ4-Anxiety subscales (score range, $0-6$ for each subscale) indicate greater depression and anxiety symptoms, respectively.

\section{Clinical Outcomes}

We investigated clinical outcomes, including hospital LOS, unplanned hospital readmissions, and overall survival. We defined hospital LOS as the number of days from admission to discharge. To determine the risk of hospital readmission, we identified unplanned readmissions within 90 days of hospital discharge and calculated the time to first unplanned readmission as the number of days from hospital discharge to the first unplanned admission within 90 days. To account for early mortality, we created a composite dichotomous outcome categorizing patients as either alive and with no readmission within 90 days or dead and/or readmitted within 90 days, consistent with prior work. ${ }^{16}$ We calculated survival as the number of days from hospital
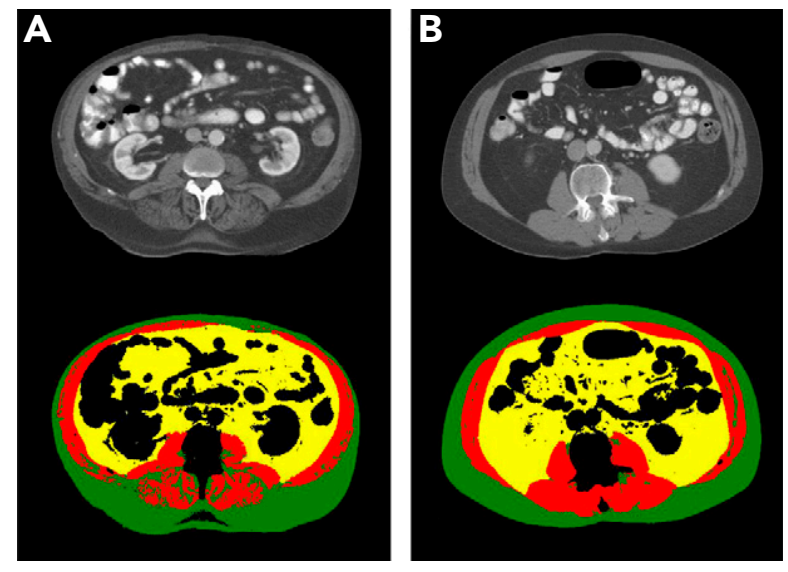

Figure 1. Axial CT images of the third lumbar vertebral region illustrating patients with (A) low and (B) high muscle radiodensity, with skeletal muscle highlighted in red, subcutaneous adipose tissue highlighted in green, and visceral adipose tissue highlighted in yellow. Patients were matched for age, sex, intravenous contrast, muscle cross-sectional area, and body mass index (tolerance: $+10 \%$ ).

discharge to date of death, censoring patients who were alive at their last follow-up date.

\section{Statistical Analysis}

We used descriptive statistics to evaluate frequencies, means, and standard deviations for participants' demographic and clinical information. To explore relationships between participant characteristics and muscle, we used multivariable linear regression, including patient age, sex, race, marital status, education level, insurance type, CCI score, time since cancer diagnosis, cancer type, BMI, and presence of intravenous contrast on the CT scan. We used linear regression to examine associations between muscle and continuous outcomes of patients' physical and psychological symptom burden as well as hospital LOS. We used Cox regression to investigate relationships between muscle and the outcomes of time to readmission within 90 days and overall survival. To evaluate the association between muscle and the dichotomized outcome of readmission or death within 90 days, we used logistic regression. In regression models, we adjusted for potential confounders, including age, sex, marital status, education level, insurance type, cancer type, BMI, and presence of intravenous contrast on the CT scan. ${ }^{9,36}$

\section{Results}

\section{Participant Sample}

Of the 1,121 patients who enrolled and completed the symptom questionnaire, $890(79.4 \%)$ had a CT scan within 45 days before enrollment. Of these 890 patients, 168 (18.9\%) did not have a CT scan covering the L3 level. 


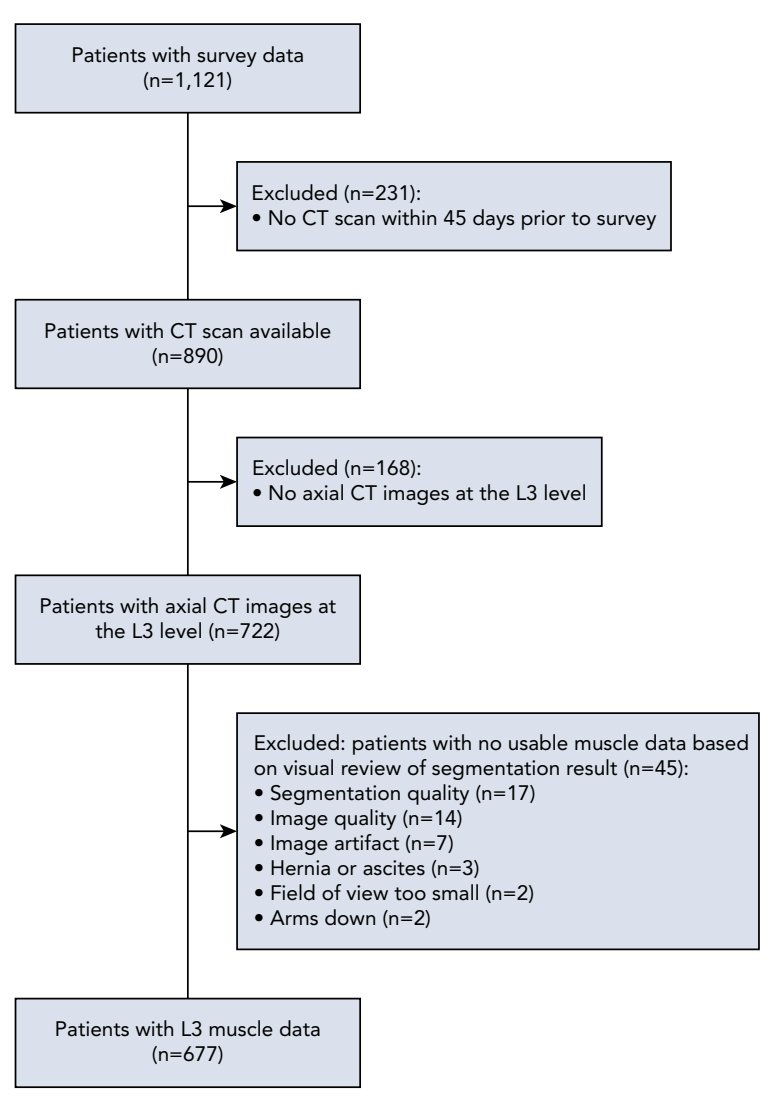

Figure 2. Patient selection flow diagram. Abbreviation: L3, third lumbar vertebral body.

Of the 722 remaining patients, 45 (6.2\%) did not have usable muscle data based on visual review of segmentation output, leaving 677 patients with evaluable muscle data at the L3 vertebral landmark (Figure 2). Participants had a mean age of $62.86 \pm 12.95$ years, and $51.1 \%$ were women (Table 1 ). In addition, participants were primarily White $(92.2 \%)$, married $(66.6 \%)$, and educated beyond high school (59.2\%). The most common cancer types were gastrointestinal (36.8\%), lung (16.7\%), and genitourinary (8.9\%). Mean time since cancer diagnosis was $38.77 \pm 51.57$ months, and mean BMI was $25.90 \pm 6.03 \mathrm{~kg} / \mathrm{m}^{2}$. Most participants $(74.4 \%)$ had CT scans with intravenous contrast. Mean hospital LOS was $6.49 \pm 4.98$ days, and the 90-day readmission and death rates were $48.9 \%$ and $40.2 \%$, respectively.

\section{Skeletal Muscle}

Patients had a mean muscle mass of $43.61 \pm 8.69 \mathrm{~cm}^{2} / \mathrm{m}^{2}$, with $64.0 \%$ of participants meeting the criteria for sarcopenia (supplemental eTable 1, available with this article at JNCCN.org). The average muscle radiodensity was $33.31 \pm 10.61 \mathrm{HU}$. We found that older age $(\mathrm{B},-0.163$; $95 \%$ CI, -0.214 to $-0.112 ; P<.001)$ and female sex (B, -6.894 ; $95 \% \mathrm{CI},-8.047$ to $-5.741 ; P<.001)$ were associated with

\section{Table 1. Patient Characteristics ( $N=677)$}

\begin{tabular}{|c|c|}
\hline Characteristic & n (\%) \\
\hline Age, mean (SD), y & $62.86(12.95)$ \\
\hline \multicolumn{2}{|l|}{ Sex } \\
\hline Female & $346(51.1)$ \\
\hline Male & 331 (48.9) \\
\hline \multicolumn{2}{|l|}{ Race } \\
\hline White & $624(92.2)$ \\
\hline Black/African American & $28(4.1)$ \\
\hline Asian & $13(1.9)$ \\
\hline Hispanic & $10(1.5)$ \\
\hline American Indian/Alaskan Native & $1(0.1)$ \\
\hline Native Hawaiian/other Pacific Islander & $1(0.1)$ \\
\hline \multicolumn{2}{|l|}{ Relationship status } \\
\hline Married & $451(66.6)$ \\
\hline Single & $111(16.4)$ \\
\hline Divorced & $76(11.2)$ \\
\hline Widowed & $39(5.8)$ \\
\hline \multicolumn{2}{|l|}{ Education level } \\
\hline High school and below & $214(31.6)$ \\
\hline Beyond high school & $401(59.2)$ \\
\hline Declined to provide & $62(9.2)$ \\
\hline \multicolumn{2}{|l|}{ Insurance type } \\
\hline Government-sponsored & $326(48.2)$ \\
\hline Private & $347(51.3)$ \\
\hline None & $4(0.6)$ \\
\hline \multicolumn{2}{|l|}{ Cancer type } \\
\hline Gastrointestinal & 249 (36.8) \\
\hline Lung & $113(16.7)$ \\
\hline Genitourinary & $60(8.9)$ \\
\hline Skin & $59(8.7)$ \\
\hline Breast & $47(6.9)$ \\
\hline Gynecologic & $36(5.3)$ \\
\hline Sarcoma & $34(5.0)$ \\
\hline Head and neck & $27(4.0)$ \\
\hline Leukemia & $22(3.2)$ \\
\hline Lymphoma & $19(2.8)$ \\
\hline Cancer of unknown primary & $11(1.6)$ \\
\hline Months since cancer diagnosis, mean (SD) & $38.77(51.57)$ \\
\hline $\mathrm{CCl}$ score, mean (SD) & $0.78(1.22)$ \\
\hline Body mass index, mean (SD), kg/m² & $25.90(6.03)$ \\
\hline CT with intravenous contrast & $504(74.4)$ \\
\hline
\end{tabular}

Abbreviation: $\mathrm{CCl}$, Charlson comorbidity index.

lower muscle mass, and higher BMI (B, 0.577; 95\% CI, $0.488-0.665 ; P<.001$ ) was associated with greater muscle mass. For muscle radiodensity, we found that older age 
(B, $-0.328 ; 95 \% \mathrm{CI},-0.386$ to $-0.270 ; P<.001$ ), female sex (B, $-1.660 ; 95 \% \mathrm{CI},-2.977$ to $-0.082 ; P=.014$ ), having a breast cancer diagnosis $(\mathrm{B},-3.391 ; 95 \% \mathrm{CI},-6.408$ to $-0.373 ; P=.028)$, and higher BMI (B, -0.608 ; 95\% CI, -0.709 to $-0.507 ; P<.001)$ were associated with lower muscle radiodensity, and education beyond high school (B, 1.881; 95\% CI, 0.637-3.125; $P=.003$ ) was associated with greater muscle radiodensity (Table 2).

\section{Relationship Between Muscle and Symptom Burden}

We found that the associations between muscle mass and patients' physical and psychological symptom burden did not reach statistical significance (Table 3, supplemental eTable 2). However, higher muscle radiodensity was significantly associated with lower physical (ESAS-Physical: B, -0.165 ; $95 \%$ CI, -0.299 to $-0.030 ; P=.016$ ) and total (ESASTotal: $\mathrm{B},-0.286$; $95 \% \mathrm{CI},-0.462$ to $-0.109 ; P=.002)$ symptom burden and with lower depression (PHQ-4-Depression: $\mathrm{B}$, -0.028 ; $95 \%$ CI, -0.048 to $-0.008 ; P=.006$ ) and anxiety (PHQ4-Anxiety: $\mathrm{B},-0.028$; $95 \% \mathrm{CI},-0.048$ to $-0.007 ; P=.008$ ).

\section{Relationship Between Muscle and Clinical Outcomes} We found that muscle mass was not significantly associated with hospital LOS or readmissions (Table 3).
However, higher muscle radiodensity was significantly associated with decreased hospital LOS (B, $-0.069 ; 95 \%$ CI, -0.117 to $-0.021 ; P=.005)$ and lower risk of readmission or death in 90 days (odds ratio, 0.966; 95\% CI, $0.945-0.986$; $P<.001)$. Neither muscle mass nor radiodensity was associated with time to readmission within 90 days. We found that both higher muscle mass (hazard ratio, 0.969; 95\% CI, 0.955-0.982; $P<.001$ ) and higher muscle radiodensity (hazard ratio, 0.969; 95\% CI, 0.958-0.981; $P<.001$ ) were significantly associated with a lower hazard for death.

\section{Discussion}

In this study of hospitalized patients with advanced cancer, we demonstrated that a substantial proportion of patients have low muscle mass consistent with sarcopenia, and we identified patient characteristics associated with muscle mass (quantity) and radiodensity (quality). Notably, while patients' muscle mass only significantly correlated with survival, we found that patients' muscle radiodensity was significantly associated with their physical and psychological symptoms, healthcare utilization, and survival outcomes. Collectively, these findings expand upon existing evidence showing unfavorable

\section{Table 2. Factors Associated With Higher Skeletal Muscle Mass and Radiodensity}

\begin{tabular}{|c|c|c|c|c|}
\hline \multirow[b]{2}{*}{ Variable } & \multicolumn{2}{|c|}{ Muscle Mass } & \multicolumn{2}{|c|}{ Muscle Radiodensity } \\
\hline & B $(95 \% \mathrm{Cl})$ & $P$ Value & B $(95 \% \mathrm{Cl})$ & $P$ Value \\
\hline Age & $-0.163(-0.214$ to -0.112$)$ & $<.001$ & $-0.328(-0.386$ to -0.270$)$ & $<.001$ \\
\hline Female sex & $-6.894(-8.047$ to -5.741$)$ & $<.001$ & $-1.660(-2.977$ to -0.082$)$ & .014 \\
\hline White race & $0.159(-1.807$ to 2.126$)$ & .874 & $-1.121(-3.367$ to 1.124$)$ & .327 \\
\hline Married & $-1.219(-2.362$ to 0.075$)$ & .037 & $0.417(-0.889$ to 1.722$)$ & .531 \\
\hline Education beyond high school & $0.341(-0.749$ to 1.430$)$ & .539 & $1.881(0.637$ to 3.125$)$ & .003 \\
\hline Government-sponsored insurance & $-0.668(-1.940$ to 0.604$)$ & .303 & $-1.393(-2.846$ to 0.059$)$ & .060 \\
\hline $\mathrm{CCl}$ score & $0.334(-0.126$ to 0.793$)$ & .154 & $-0.101(-0.626$ to 0.423$)$ & .705 \\
\hline Months since cancer diagnosis & $-0.004(-0.016$ to 0.007$)$ & .474 & $-0.003(-0.016$ to 0.011$)$ & .707 \\
\hline \multicolumn{5}{|l|}{ Cancer type } \\
\hline Gastrointestinal & $0.338(-1.440$ to 2.116$)$ & .709 & $-0.368(-2.398$ to 1.662$)$ & .722 \\
\hline Lung & $-0.458(-2.485$ to 1.568$)$ & .657 & $-0.021(-2.336$ to 2.293$)$ & .986 \\
\hline Hematologic & $0.443(-2.248$ to 3.134$)$ & .747 & $-0.938(-4.010$ to 2.135$)$ & .549 \\
\hline Genitourinary & $0.600(-1.854$ to 3.053$)$ & .632 & $-1.131(-3.933$ to 1.671$)$ & .428 \\
\hline Breast & $-0.942(-3.585$ to 1.701$)$ & .484 & $-3.391(-6.408$ to -0.373$)$ & .028 \\
\hline Skin & $0.599(-1.792$ to 2.990$)$ & .623 & $1.212(-1.519$ to 3.942$)$ & .384 \\
\hline Head and neck & $-0.216(-3.288$ to 2.855$)$ & .890 & $-0.152(-3.659$ to 3.355$)$ & .932 \\
\hline Other ${ }^{a}$ & - & - & - & - \\
\hline Body mass index & $0.577(0.488$ to 0.665$)$ & $<.001$ & $-0.608(-0.709$ to -0.507$)$ & $<.001$ \\
\hline CT with intravenous contrast & $1.397(0.161$ to 2.633$)$ & .027 & $3.228(1.816$ to 4.639$)$ & $<.001$ \\
\hline
\end{tabular}

Bold indicates statistically significant values.

Abbreviations: $\mathrm{B}$, unstandardized coefficient; $\mathrm{CCl}$, Charlson comorbidity index.

ancludes sarcoma, gynecologic malignancies, and cancer of unknown primary. 
Table 3. Relationships Between Skeletal Muscle and Patient Outcomes

\begin{tabular}{|c|c|c|c|c|}
\hline Patient-Reported Outcomes ${ }^{a}$ & \multicolumn{2}{|l|}{ Muscle Mass } & \multicolumn{2}{|c|}{ Muscle Radiodensity } \\
\hline ESAS-Physical & $-0.101(-0.254$ to 0.053$)$ & .198 & $-0.165(-0.299$ to -0.030$)$ & .016 \\
\hline PHQ-4-Depression & $-0.015(-0.037$ to 0.007$)$ & .185 & $-0.028(-0.048$ to -0.008$)$ & .006 \\
\hline PHQ-4-Anxiety & $-0.009(-0.032$ to 0.014$)$ & .437 & $-0.028(-0.048$ to -0.007$)$ & .008 \\
\hline Hospital LOS (B) & $-0.048(-0.103$ to 0.007$)$ & .088 & $-0.069(-0.117$ to -0.021$)$ & .005 \\
\hline $\begin{array}{l}\text { Readmission/Death in } 90 \text { days } \\
\text { (OR) }\end{array}$ & 0.988 (0.964 to 1.012$)$ & .309 & 0.966 (0.945 to 0.986$)$ & $<.001$ \\
\hline $\begin{array}{l}\text { Time to readmission in } 90 \text { days } \\
\text { (HR) }\end{array}$ & 1.006 (0.989 to 1.024$)$ & .460 & 0.989 (0.975 to 1.004$)$ & .145 \\
\hline Time to death (HR) & 0.969 (0.955 to 0.982 ) & $<.001$ & 0.969 (0.958 to 0.981$)$ & $<.001$ \\
\hline
\end{tabular}

Bold indicates statistically significant values.

Abbreviations: B, unstandardized coefficient; ESAS, Edmonton Symptom Assessment System; HR, hazard ratio; LOS, length of stay; OR, odds ratio; PHQ-4, Patient Health Questionnaire-4.

aModels adjusted for age, sex, marital status, education level, insurance type, cancer type, body mass index, and use of intravenous contrast for the CT scan

outcomes associated with poor muscle health in patients with cancer. Importantly, this work further underscores the added utility of assessing patients' muscle radiodensity when seeking to address adverse muscle changes in oncology, including high symptom burden, increased healthcare utilization, and poor survival.

Our work highlights the importance of assessing and addressing muscle health among hospitalized patients with cancer. More than $60 \%$ of patients met the criteria for sarcopenia, which is higher than in prior reports and may relate to the fact that our cohort consisted of patients with unplanned hospitalizations, a population particularly vulnerable to muscle loss. ${ }^{9}$ A more complete understanding of muscle health in oncology could inform future work, such as (1) investigating potential mechanisms for how low muscle mass and radiodensity impact patient outcomes, (2) identifying patients at risk for experiencing a decrease in muscle mass or radiodensity, and (3) developing interventions targeting adverse muscle changes in this population. Our work presents compelling new evidence supporting the need to address muscle health among patients with cancer, particularly muscle radiodensity, to enhance outcomes in this population.

This study is the first to report that hospitalized patients' muscle radiodensity, as assessed on routine clinical CT scans, is associated with their physical and psychological symptom burden. Hospitalized patients represent a uniquely vulnerable population at increased risk for high symptom burden, but studies investigating the relationship between these patients' muscle health and symptom burden are lacking. ${ }^{18,28,31}$ Interestingly, we found significant relationships between patients' muscle radiodensity and their symptom burden, but did not observe significant associations between muscle mass and symptom burden. One potential explanation linking muscle radiodensity with symptom burden is that low muscle radiodensity may lead to functional decline more so than low muscle mass alone, and thereby exacerbate physical and psychological symptoms. ${ }^{13,21}$ Conversely, patients with a higher symptom burden may have lower physical activity, which could have differential effects on their muscle radiodensity and mass. ${ }^{21,42,43}$ Ultimately, these findings are hypothesisgenerating and merit confirmation in future studies to better understand potential underlying mechanisms and to investigate the differential effects of muscle mass and radiodensity on patients' functional, clinical, and patientreported outcomes.

Importantly, we also found significant relationships between patients' muscle health and their clinical outcomes, namely, healthcare utilization and survival. Although prior work has shown that muscle mass correlates with survival, limited data exist regarding healthcare utilization, and even fewer studies have examined muscle radiodensity in relation to these outcomes. ${ }^{6,7}$ Clinically, muscle loss in patients with cancer often corresponds with worsening physical function, increased disease burden, and poor treatment tolerance, all of which can contribute to high healthcare utilization. ${ }^{44,45}$ We found that lower muscle radiodensity was associated with longer hospital LOS and higher risk of death or readmission following hospital discharge, but muscle mass did not show significant associations with these outcomes. Plausibly, muscle radiodensity identifies a population with high symptom burden and greater risk for needing hospitallevel care, as shown by our findings that muscle radiodensity, and not muscle mass, was significantly associated 
with symptom burden. ${ }^{28}$ Notably, we found that both muscle radiodensity and mass are associated with survival, a finding that aligns with extensive prior literature among patients with cancer in outpatient settings. ${ }^{2,6,7,35,46}$ Our data expand on prior data supporting muscle health as an important prognostic indicator, and we show that muscle radiodensity represents a significant predictor of high healthcare utilization. Thus, future work should consider prospective comprehensive muscle assessment as a strategy for identifying patients who may benefit from innovative care models, such as fitness and/or nutrition interventions, to enhance clinical outcomes.

In addition, we identified patient characteristics associated with poor muscle health. Consistent with prior work, patients who were older and female had lower muscle mass and radiodensity, potentially related to age- and sex-specific differences in neurologic, hormonal, nutritional, metabolic, and physical function factors. ${ }^{19,25,47-49}$ We also found that patients with breast cancer had lower muscle radiodensity compared with other cancer types, which is hypothesisgenerating and may relate to our finding of lower muscle radiodensity in female patients. Interestingly, we found that higher BMI was associated with higher muscle mass, yet conversely with lower muscle radiodensity. By definition, low muscle radiodensity correlates with greater intramuscular adipose tissue, and thus higher BMI may contribute to fatty infiltration of muscle tissue. ${ }^{15}$ Furthermore, the relationship between higher muscle mass and higher BMI may reflect the fact that greater muscle mass adds to body weight and thereby increases BMI. This detail may also play a role in the "obesity paradox" concept, a phenomenon in which patients with higher BMI experience favorable survival outcomes in oncology. ${ }^{6,50,51}$ Notably, radiodensity may play a role in the obesity paradox by allowing clinicians to further characterize the muscle health of patients, regardless of their muscle mass and BMI. Moreover, overlap likely exists between muscle mass and radiodensity, and research has increasingly begun to look at the different body composition phenotypes that confer additional risk of poor outcomes. ${ }^{1,13}$ By identifying patient characteristics associated with low muscle mass and radiodensity, our findings can inform efforts to detect individuals at greater risk of experiencing adverse muscle changes who may benefit from targeted supportive care interventions.

Several limitations warrant discussion. First, we conducted this study at a single, tertiary care site in a population with limited sociodemographic diversity. Therefore, the study may not generalize to more heterogeneous populations. Second, we analyzed skeletal muscle at a single cross-section of time, and we lack data about longitudinal changes in patients' muscle mass and radiodensity. Thus, we cannot determine the direction of the associations we observed or determine the potential mechanisms underlying these associations. Third, we investigated a heterogeneous group of patients with various cancer types who likely experience variable muscle changes. ${ }^{46}$ However, we sought to minimize this variation by including only hospitalized patients with incurable disease and adjusted for cancer type in our multivariable models. Fourth, we lack information about other potentially important factors that could influence patients' muscle health, symptom burden, and clinical outcomes, such as physical and mental function, coping strategies, and quality of social supports. Fifth, we used CT scans to assess muscle mass and radiodensity given that they are often obtained as part of routine clinical care, yet other methodologies to examine muscle radiodensity exist, including ultrasound and MRI. ${ }^{52-55}$ Finally, by using a 45-day window before enrollment for CT scans assessing muscle, we may be underestimating the degree of association between patients' muscle health and their patient-reported and clinical outcomes.

\section{Conclusions}

This study provides innovative findings demonstrating the prevalence and predictors of poor muscle health among hospitalized patients with advanced cancer. We found significant relationships between skeletal muscle, patient-reported symptom burden, and clinical outcomes in a large cohort of hospitalized patients with advanced cancer. Specifically, we observed that muscle mass (quantity) only correlated with patients' survival, while muscle radiodensity (quality) demonstrated significant relationships with patients' symptom burden, healthcare utilization, and survival. Collectively, these findings underscore the need to assess and address muscle health among patients with cancer, while also highlighting the added importance of muscle radiodensity as a predictor of both patient-reported and clinical outcomes. Future studies should seek to develop and test interventions addressing muscle health in patients with advanced cancer, while also evaluating the impact of such interventions on physical and psychological symptom burden, healthcare utilization, and survival outcomes.

Submitted April 23, 2020; accepted for publication July 8, 2020. Published online January 29, 2021.

Previous presentation: This study was presented at the 2020 ASCO Virtual Scientific Program; May 29-31, 2020. Abstract 7006.

Author contributions: Study concept and design: All authors. Data acquisition, analysis and interpretation: All authors. Manuscript preparation: All authors. Critical revision: All authors. Final approval of manuscript: All authors. 
Disclosures: Dr. Roeland has disclosed that he receives consulting fees from Mitobridge Inc., DRG Consulting, Asahi Kasei Pharmaceuticals, Napo Pharmaceuticals, American Imaging Management, Immuneering Corporation, and Prime Oncology; is a scientific advisor for Heron Pharmaceuticals, Vector Oncology, and Helsinn Pharmaceuticals; and has served as a member on data safety monitoring boards for Oragenics, Inc., Galera Pharmaceuticals, and Enzychem Lifesciences Pharmaceutical Company. Dr. Fintelmann has disclosed that he has received royalties from Massachusetts General/ Brigham \& Women's. The remaining authors have disclosed that they have not received any financial consideration from any person or organization to support the preparation, analysis, results, or discussion of this article.

Funding: Research reported in this publication was supported by the $\mathrm{NCl}$ of the NIH under award number K24 CA181253.

Disclaimer: The content is solely the responsibility of the authors and does not necessarily represent the official views of the $\mathrm{NIH}$.

Correspondence: Ryan Nipp, MD, MPH, Department of Medicine, Division of Hematology and Oncology, Massachusetts General Hospital Cancer Center and Harvard Medical School, 55 Fruit Street, Yawkey Center, Suite 7E, Boston, MA 02114. Email: RNipp@MGH.Harvard.edu

\section{References}

1. Weinberg MS, Shachar SS, Muss HB, et al. Beyond sarcopenia: characterization and integration of skeletal muscle quantity and radiodensity in a curable breast cancer population. Breast J 2018;24:278-284.

2. Kroenke $\mathrm{CH}$, Prado $\mathrm{CM}$, Meyerhardt JA, et al. Muscle radiodensity and mortality in patients with colorectal cancer. Cancer 2018;124: 3008-3015.

3. Inagaki J, Rodriguez V Bodey GP. Proceedings: causes of death in cancer patients. Cancer 1974;33:568-573.

4. Tisdale MJ. Biology of cachexia. J Natl Cancer Inst 1997;89:1763-1773.

5. Fearon KC. Cancer cachexia: developing multimodal therapy for a multidimensional problem. Eur J Cancer 2008;44:1124-1132.

6. Caan BJ, Meyerhardt JA, Kroenke $\mathrm{CH}$, et al. Explaining the obesity paradox: the association between body composition and colorecta cancer survival (C-SCANS study). Cancer Epidemiol Biomarkers Prev 2017;26:1008-1015

7. Deluche E, Leobon S, Desport JC, et al. Impact of body composition on outcome in patients with early breast cancer. Support Care Cancer 2018; 26:861-868

8. Bruggeman AR, Kamal AH, LeBlanc TW, et al. Cancer cachexia: beyond weight loss. J Oncol Pract 2016;12:1163-1171.

9. Nipp RD, Fuchs G, El-Jawahri A, et al. Sarcopenia is associated with quality of life and depression in patients with advanced cancer. Oncologist 2018;23:97-104

10. Tsaousi G, Kokkota S, Papakostas P, et al. Body composition analysis for discrimination of prolonged hospital stay in colorectal cancer surgery patients. Eur J Cancer Care (Engl) 2017;26:e12491.

11. Miljkovic I, Zmuda JM. Epidemiology of myosteatosis. Curr Opin Clin Nutr Metab Care 2010;13:260-264.

12. Looijaard WGPM, Dekker IM, Stapel SN, et al. Skeletal muscle quality as assessed by CT-derived skeletal muscle density is associated with 6-month mortality in mechanically ventilated critically ill patients. Crit Care 2016;20:386

13. Williams GR, Deal AM, Muss HB, et al. Skeletal muscle measures and physical function in older adults with cancer: sarcopenia or myopenia? Oncotarget 2017;8:33658-33665.

14. Martin L, Birdsell L, Macdonald N, et al. Cancer cachexia in the age of obesity: skeletal muscle depletion is a powerful prognostic factor, independent of body mass index. J Clin Oncol 2013;31:1539-1547.

15. Stretch C, Aubin JM, Mickiewicz B, et al. Sarcopenia and myosteatosis are accompanied by distinct biological profiles in patients with pancreatic and periampullary adenocarcinomas. PLoS One 2018;13:e0196235.

16. Lage DE, Nipp RD, D'Arpino SM, et al. Predictors of posthospital transitions of care in patients with advanced cancer. J Clin Oncol 2018;36: 76-82.

17. Nipp RD, El-Jawahri A, D'Arpino SM, et al. Symptoms of posttraumatic stress disorder among hospitalized patients with cancer. Cancer 2018; 124:3445-3453.

18. Portenoy RK, Thaler HT, Kornblith AB, et al. The Memorial Symptom Assessment Scale: an instrument for the evaluation of symptom prevalence, characteristics and distress. Eur J Cancer 1994;30:1326-1336.

19. Morishita S, Kaida K, Tanaka T, et al. Prevalence of sarcopenia and relevance of body composition, physiological function, fatigue, and healthrelated quality of life in patients before allogeneic hematopoietic stem cell transplantation. Support Care Cancer 2012;20:3161-3168.

20. Wolfe RR. The underappreciated role of muscle in health and disease. Am J Clin Nutr 2006;84:475-482.

21. Keogh JWL, MacLeod RD. Body composition, physical fitness, functional performance, quality of life, and fatigue benefits of exercise for prostate cancer patients: a systematic review. J Pain Symptom Manage 2012;43: 96-110.

22. Cawthon PM, Fox KM, Gandra SR, et al. Do muscle mass, muscle density, strength, and physical function similarly influence risk of hospitalization in older adults? J Am Geriatr Soc 2009:57:1411-1419.

23. Zhou T, Yang K, Thapa S, et al. Differences in symptom burden among cancer patients with different stages of cachexia. J Pain Symptom Manage 2017:53:919-926.

24. Tsekoura M, Kastrinis A, Katsoulaki M, et al. Sarcopenia and its impact on quality of life. Adv Exp Med Biol 2017;987:213-218.

25. Fearon K, Strasser F, Anker SD, et al. Definition and classification of cancer cachexia: an international consensus. Lancet Oncol 2011:12: 489-495.

26. Basch E, Deal AM, Dueck AC, et al. Overall survival results of a trial assessing patient-reported outcomes for symptom monitoring during routine cancer treatment. JAMA 2017;318:197-198.

27. Basch E, Deal AM, Kris MG, et al. Symptom monitoring with patientreported outcomes during routine cancer treatment: a randomized controlled trial. J Clin Oncol 2016;34:557-565.

28. Nipp RD, El-Jawahri A, Moran SM, et al. The relationship between physical and psychological symptoms and health care utilization in hospitalized patients with advanced cancer. Cancer 2017;123: 4720-4727.

29. Teunissen SCCM, Wesker W, Kruitwagen C, et al. Symptom prevalence in patients with incurable cancer: a systematic review. J Pain Symptom Manage 2007;34:94-104.

30. Berry DL. Patient-reported symptoms and quality of life integrated into clinical cancer care. Semin Oncol Nurs 2011;27:203-210.

31. van Seventer EE, Fintelmann FJ, Roeland EJ, et al. Leveraging the potential synergy between patient-reported outcomes and body composition analysis in patients with cancer. Oncologist 2020;25:271-273.

32. Johnson PC, Xiao Y, Wong RL, et al. Potentially avoidable hospital readmissions in patients with advanced cancer. J Oncol Pract 2019;15: e420-427.

33. El-Jawahri A, Lau-Min K, Nipp RD, et al. Processes of code status transitions in hospitalized patients with advanced cancer. Cancer 2017;123: 4895-4902.

34. Bridge $C P$, Rosenthal M, Wright $B$, et al. Fully-automated analysis of body composition from $\mathrm{CT}$ in cancer patients using convolutional neural networks. In: Stoyanov D, Taylor Z, Sarikaya D, et al, eds. OR 2.0 Context-Aware Operating Theaters, Computer Assisted Robotic Endoscopy, Clinical Image-Based Procedures, and Skin Image Analysis. Basel, Switzerland: Springer International Publishing; 2018:204-213.

35. Troschel AS, Troschel FM, Best TD, et al. Computed tomography-based body composition analysis and its role in lung cancer care. J Thorac Imaging 2020;35:91-100.

36. Fuchs G, Chretien YR, Mario J, et al. Quantifying the effect of slice thickness, intravenous contrast and tube current on muscle segmentation: implications for body composition analysis. Eur Radiol 2018;28: 2455-2463.

37. Bruera E, Kuehn N, Miller MJ, et al. The Edmonton Symptom Assessment System (ESAS): a simple method for the assessment of palliative care patients. J Palliat Care 1991;7:6-9.

38. Chang VT, Hwang SS, Feuerman M. Validation of the Edmonton Symptom Assessment Scale. Cancer 2000;88:2164-2171.

39. Hui D, Bruera E. The Edmonton Symptom Assessment System 25 years later: past, present, and future developments. J Pain Symptom Manage 2017:53:630-643 
40. Rhondali W, Nguyen L, Palmer L, et al. Self-reported constipation in patients with advanced cancer: a preliminary report. J Pain Symptom Manage 2013;45:23-32.

41. Kroenke K, Spitzer RL, Williams JBW, et al. An ultra-brief screening scale for anxiety and depression: the PHQ-4. Psychosomatics 2009;50: 613-621.

42. Müller $\mathrm{V}$, Nabieva $\mathrm{N}$, Häberle $\mathrm{L}$, et al. Impact of disease progression on health-related quality of life in patients with metastatic breast cancer in the PRAEGNANT breast cancer registry. Breast 2018;37:154-160.

43. Gonzalez-Saenz de Tejada M, Bilbao A, Baré M, et al. Association between social support, functional status, and change in health-related quality of life and changes in anxiety and depression in colorectal cancer patients. Psychooncology 2017;26:1263-1269.

44. Dunne RF, Loh KP, Williams GR, et al. Cachexia and sarcopenia in older adults with cancer: a comprehensive review. Cancers (Basel) 2019;11: 1861.

45. Prado CM, Purcell SA, Alish $C$, et al. Implications of low muscle mass across the continuum of care: a narrative review. Ann Med 2018;50: 675-693.

46. Ryan AM, Power DG, Daly L, et al. Cancer-associated malnutrition, cachexia and sarcopenia: the skeleton in the hospital closet 40 years later. Proc Nutr Soc 2016;75:199-211.

47. Gewandter JS, Dale W, Magnuson A, et al. Associations between a patient-reported outcome (PRO) measure of sarcopenia and falls, functional status, and physical performance in older patients with cancer J Geriatr Oncol 2015;6:433-441.

48. Doherty TJ. Invited review: aging and sarcopenia. J Appl Physiol (1985) 2003;95:1717-1727

49. Anderson LJ, Liu H, Garcia JM. Sex differences in muscle wasting. Adv Exp Med Biol 2017;1043:153-197.

50. Kichenadasse G, Miners JO, Mangoni AA, et al. Association between body mass index and overall survival with immune checkpoint inhibitor therapy for advanced non-small cell lung cancer. JAMA Oncol 2020;7: 512-518.

51. Strulov Shachar S, Williams GR. The obesity paradox in cancer-moving beyond BMI. Cancer Epidemiol Biomarkers Prev 2017;26:13-16.

52. van Dijk DPJ, Bakers FCH, Sanduleanu S, et al. Myosteatosis predicts survival after surgery for periampullary cancer: a novel method using MRI HPB (Oxford) 2018:20:715-720.

53. Aubrey J, Esfandiari N, Baracos VE, et al. Measurement of skeletal muscle radiation attenuation and basis of its biological variation. Acta Physiol (Oxf) 2014;210:489-497.

54. Mitsiopoulos N, Baumgartner RN, Heymsfield SB, et al. Cadaver validation of skeletal muscle measurement by magnetic resonance imaging and computerized tomography. J Appl Physiol (1985) 1998;85: 115-122.

55. Heymsfield SB, Gonzalez MC, Lu J, et al. Skeletal muscle mass and quality: evolution of modern measurement concepts in the context of sarcopenia. Proc Nutr Soc 2015;74:355-366. 
Supplemental online content for:

\section{Associations of Skeletal Muscle With Symptom Burden and Clinical Outcomes in Hospitalized Patients With Advanced Cancer}

Emily van Seventer, MPH; J. Peter Marquardt; Amelie S. Troschel; Till D. Best; Nora Horick, MS; Chinenye Azoba, BS; Richard Newcomb, MD; Eric J. Roeland, MD; Michael Rosenthal, MD, PhD; Christopher P. Bridge, PhD; Joseph A. Greer, PhD; Areej El-Jawahri, MD; Jennifer Temel, MD; Florian J. Fintelmann, MD; and Ryan D. Nipp, MD, MPH

J Natl Compr Canc Netw 2021;19(3):319-327

eTable 1: Body Composition Metrics Stratified by Age, Sex, Sarcopenia, BMI, and Sarcopenic Obesity eTable 2: Relationships Between Skeletal Muscle and Patient Outcomes 


\begin{tabular}{|c|c|c|c|c|}
\hline Characteristic & $\mathbf{n}$ & $\begin{array}{c}\text { Muscle Mass, } \mathrm{cm}^{2} / \mathrm{m}^{2} \\
\text { Mean (SD) }\end{array}$ & $\begin{array}{l}\text { Muscle Radiodensity, HU } \\
\text { Mean (SD) }\end{array}$ & $\begin{array}{l}\text { Sarcopenia } \\
\text { n (\%) }\end{array}$ \\
\hline \multicolumn{5}{|l|}{ Age, y } \\
\hline$<65$ & 373 & $45.06(9.02)$ & $36.88(9.64)$ & $211(56.6)$ \\
\hline Female & 331 & $40.14(7.30)$ & $32.65(10.16)$ & $151(45.6)$ \\
\hline Male & 346 & $46.93(8.64)$ & $33.94(10.13)$ & $282(81.5)$ \\
\hline \multicolumn{5}{|l|}{ Sarcopenic } \\
\hline Yes & 433 & $40.53(7.18)$ & $32.54(10.29)$ & - \\
\hline $18.50-24.99$ & 299 & $41.14(6.76)$ & $35.86(9.06)$ & $224(74.9)$ \\
\hline $25-29.99$ & 198 & $46.10(9.11)$ & $31.82(10.34)$ & $118(59.6)$ \\
\hline $30-34.99$ & 85 & $47.65(8.48)$ & $30.12(9.38)$ & $36(42.4)$ \\
\hline $35-39.99$ & 27 & $49.86(9.72)$ & $26.27(8.20)$ & $8(29.6)$ \\
\hline$\geq 40$ & 22 & $48.52(10.03)$ & $21.65(10.45)$ & $7(31.8)$ \\
\hline Unknown weight & 2 & $44.15(4.16)$ & $28.47(10.25)$ & $2(100)$ \\
\hline \multicolumn{5}{|l|}{ Sarcopenic obesity ${ }^{a}$} \\
\hline Yes & 51 & $43.34(8.18)$ & $26.28(11.45)$ & $51(100)$ \\
\hline No & 624 & $43.63(8.75)$ & $33.90(9.84)$ & $380(60.9)$ \\
\hline
\end{tabular}

Abbreviation: BMl, body mass index.

${ }^{a}$ Criteria for sarcopenic obesity included cross-sectional muscle area at the third lumbar vertebra of $<39 \mathrm{~cm}^{2} / \mathrm{m}^{2}$ for women and $<55 \mathrm{~cm} / \mathrm{m}^{2}$ for men with a $\mathrm{BMI} \geq 30 \mathrm{~kg} / \mathrm{m}^{2}$.

\section{eTable 2. Relationships Between Skeletal Muscle and Patient Outcomes}

\begin{tabular}{|c|c|c|c|c|}
\hline Patient-Reported Outcomes ${ }^{a}$ & \multicolumn{2}{|l|}{ Muscle Mass } & \multicolumn{2}{|c|}{ Muscle Radiodensity } \\
\hline ESAS-Physical & $-0.033(-0.199$ to 0.133$)$ & .699 & $-0.153(-0.299$ to -0.008$)$ & .039 \\
\hline PHQ-4-Depression & $-0.003(-0.027$ to 0.022$)$ & .818 & $-0.027(-0.048$ to -0.005$)$ & .016 \\
\hline PHQ-4-Anxiety & $0.004(-0.021$ to 0.029$)$ & .746 & $-0.029(-0.052$ to -0.007$)$ & .011 \\
\hline Readmission/Death in 90 days (OR) & 1.003 (0.977 to 1.029$)$ & .830 & 0.965 (0.943 to 0.987$)$ & .002 \\
\hline Time to readmission in 90 days (HR) & 1.014 (0.995 to 1.034$)$ & .139 & 0.984 (0.968 to 1.000$)$ & .053 \\
\hline Time to death (HR) & 0.980 (0.965 to 0.995$)$ & .008 & 0.977 (0.964 to 0.989$)$ & .0001 \\
\hline
\end{tabular}

Bold indicates statistically significant values.

Abbreviations: B, unstandardized coefficient; ESAS, Edmonton Symptom Assessment System; HR, hazard ratio; LOS, length of stay; OR, odds ratio; PHQ-4, Patient Health Questionnaire-4.

aModels adjusted for age, sex, marital status, education level, insurance type, cancer type, body mass index, and use of intravenous contrast for the CT scan. 